\title{
Human Papillomavirus Vaccination in LGBTQ Patients: The Need for Dermatologists on the Front Lines
}

\author{
Alexander M. Cartron, BS; John C. Trinidad, MD, MPH
}

$\mid$ uman papillomavirus (HPV) is one of the most common sexually transmitted infections in the United States. It is the causative agent of genital warts, as well as cervical, anal, penile, vulvar, vaginal, and some head and neck cancers. ${ }^{1}$ Development of the HPV vaccine and its introduction into the scheduled vaccine series recommended by the Centers for Disease Control and Prevention (CDC) represented a major public health milestone. The CDC recommends the HPV vaccine for all children beginning at 11 or 12 years of age, even as early as 9 years, regardless of gender identity or sexuality. As of late 2016, the 9-valent formulation (Gardasil 9 [Merck]) is the only HPV vaccine distributed in the United States, and the vaccination schedule depends specifically on age. The Advisory Committee on Immunization Practices (ACIP) of the CDC revised its recommendations in 2019 to include "shared clinical decision-making regarding HPV vaccination . . . for some adults aged 27 through 45 years. ${ }^{2}$ This change in policy has notable implications for sexual and gender minority populations, such as lesbian, gay, bisexual, transgender, and queer or questioning (LGBTQ) patients, especially in the context of dermatologic care. Herein, we discuss HPV-related conditions for LGBTQ patients, barriers to vaccine administration, and the role of dermatologists in promoting an increased vaccination rate in the LGBTQ community.

\section{HPV-Related Conditions}

A 2019 review of dermatologic care for LGBTQ patients identified many specific health disparities of HPV. ${ }^{3}$
Specifically, men who have sex with men (MSM) are more likely than heterosexual men to have oral, anal, and penile HPV infections, including high-risk HPV types. ${ }^{3}$ From 2011 to $2014,18 \%$ and $13 \%$ of MSM had oral HPV infection and high-risk oral HPV infection, respectively, compared to only $11 \%$ and $7 \%$, respectively, of men who reported never having had a same-sex sexual partner. ${ }^{4}$

Similarly, despite the CDC's position that patients with perianal warts might benefit from digital anal examination or referral for standard or high-resolution anoscopy to detect intra-anal warts, improvements in morbidity have not yet been realized. In 2017, anal cancer incidence was 45.9 cases for every 100,000 person-years among human immunodeficiency (HIV)-positive MSM and 5.1 cases for every 100,000 person-years among HIV-negative MSM vs only 1.5 cases for every 100,000 person-years among men in the United States overall. ${ }^{3}$ Yet the CDC states that there is insufficient evidence to recommend routine anal cancer screening among MSM, even when a patient is HIV positive. Therefore, current screening practices and treatments are insufficient as MSM continue to have a disproportionately higher rate of HPV-associated disease compared to other populations.

\section{Barriers to HPV Vaccine Administration}

The HPV vaccination rate among MSM in adolescent populations varies across reports. ${ }^{5-7}$ Interestingly, a 2016 survey study found that MSM had approximately 2-times greater odds of initiating the HPV vaccine than heterosexual men. ${ }^{8}$ However, a study specifically sampling young

Mr. Cartron is from the Department of Dermatology, University of Maryland School of Medicine, Baltimore. Dr. Trinidad is from the Division of Dermatology, Department of Internal Medicine, The Ohio State University Wexner Medical Center, Columbus.

The authors report no conflict of interest.

Correspondence: Alexander M. Cartron, BS, Department of Dermatology, University of Maryland School of Medicine, 419 W Redwood St, Ste 235, Baltimore, MD 21201 (alexander.cartron@som.umaryland.edu).

doi: $10.12788 /$ cutis.0084 
gay and bisexual men $(\mathrm{N}=428)$ found that only $13 \%$ had received any doses of the HPV vaccine. ${ }^{6}$

Regardless, HPV vaccination is much less common among all males than it is among all females, and the low rate of vaccination among sexual minority men has a disproportionate impact, given their higher risk for HPV infection. ${ }^{4}$ Although the HPV vaccination rate increased from 2014 to 2017, the HPV vaccination rate in MSM overall is less than half of the Healthy People 2020 goal of $80 \% .^{9}$ A 2018 review determined that HPV vaccination is a cost-effective strategy for preventing anal cancer in $\mathrm{MSM}^{10}$; yet male patients might still view the HPV vaccine as a "women's issue" and are less likely to be vaccinated if they are not prompted by health care providers. Additionally, HPV vaccination is remarkably less likely in MSM when patients are older, uninsured, of lower socioeconomic status, or have not disclosed their sexual identity to their health care provider. ${ }^{9}$ Dermatologists should be mindful of these barriers to promote HPV vaccination in MSM before, or soon after, sexual debut.

Other members of the LGBTQ community, such as women who have sex with women, face notable HPV-related health disparities and would benefit from increased vaccination efforts by dermatologists. Adolescent and young adult women who have sex with women are less likely than heterosexual adolescent and young adult women to receive routine Papanicolaou tests and initiate HPV vaccination, despite having a higher number of lifetime sexual partners and a higher risk for HPV exposure. ${ }^{11}$ A 2015 survey study $(\mathrm{N}=3253)$ found that after adjusting for covariates, only $8.5 \%$ of lesbians and $33.2 \%$ of bisexual women and girls who had heard of the HPV vaccine had initiated vaccination compared to $28.4 \%$ of their heterosexual counterparts. ${ }^{11}$ The HPV vaccine is an effective public health tool for the prevention of cervical cancer in these populations. A study of women aged 15 to 19 years in the HPV vaccination era (2007-2014) found significant $(P<.05)$ observed population-level decreases in cervical intraepithelial neoplasia incidence across all grades. ${ }^{12}$

Transgender women also face a high rate of HPV infection, HIV infection, and other structural and financial disparities, such as low insurance coverage, that can limit their access to vaccination. Transgender men have a higher rate of HPV infection than cisgender men, and those with female internal reproductive organs are less likely to receive routine Papanicolaou tests. A 2018 survey study found that approximately one-third of transgender men and women reported initiating the HPV vaccination series, ${ }^{13}$ but further investigation is required to make balanced comparisons to cisgender patients.

\section{The Role of the Dermatologist}

Collectively, these disparities emphasize the need for increased involvement by dermatologists in HPV vaccination efforts for all LGBTQ patients. Adult patients may have concerns about ties of the HPV vaccine to drug manufacturers and the general safety of vaccination. For pediatric patients, parents/guardians also may be concerned about an assumed but not evidencebased increase in sexual promiscuity following HPV vaccination. ${ }^{14}$ These topics can be challenging to discuss, but dermatologists have the duty to be proactive and initiate conversation about HPV vaccination, as opposed to waiting for patients to express interest. Dermatologists should stress the safety of the vaccine as well as its potential to protect against multiple, even life-threatening diseases. Providers also can explain that the ACIP recommends catch-up vaccination for all individuals through 26 years of age, regardless of sexual orientation or gender identity.

With the ACIP having recently expanded the appropriate age range for HPV vaccination, we encourage dermatologists to engage in education and shared decision-making to ensure that adult patients with specific risk factors receive the HPV vaccine. Because the expanded ACIP recommendations are aimed at vaccination before HPV exposure, vaccination might not be appropriate for all LGBTQ patients. However, eliciting a sexual history with routine patient intake forms or during the clinical encounter ensures equal access to the HPV vaccine.

Greater awareness of HPV-related disparities and barriers to vaccination in LGBTQ populations has the potential to notably decrease HPV-associated mortality and morbidity. Increased involvement by dermatologists contributes to the efforts of other specialties in universal HPV vaccination, regardless of sexual orientation or gender identity -ideally in younger age groups, such that patients receive the vaccine prior to coitarche.

There are many ways that dermatologists can advocate for HPV vaccination. Those in a multispecialty or academic practice can readily refer patients to an associated internist, primary care physician, or vaccination clinic in the same building or institution. Dermatologists in private practice might be able to administer the HPV vaccine themselves or can advocate for patients to receive the vaccine at a local facility of the Department of Health or at a nonprofit organization, such as a Planned Parenthood center. Although pediatricians and family physicians remain front-line providers of these services, dermatologists represent an additional member of a patient's care team, capable of advocating for this important intervention.

\section{REFERENCES}

1. Brianti P, De Flammineis E, Mercuri SR. Review of HPV-related diseases and cancers. New Microbiol. 2017;40:80-85.

2. Meites E, Szilagyi PG, Chesson HW, et al. Human papillomavirus vaccination for adults: updated recommendations of the Advisory Committee on Immunization Practices. MMWR Morb Mortal Wkly Rep. 2019;68:698-702.

3. Yeung H, Luk KM, Chen SC, et al. Dermatologic care for lesbian, gay, bisexual, and transgender persons: epidemiology, screening, and disease prevention. J Am Acad Dermatol. 2019;80:591-602. 
4. Sonawane K, Suk R, Chiao EY, et al. Oral human papillomavirus infection: differences in prevalence between sexes and concordance with genital human papillomavirus infection, NHANES 2011 to 2014. Ann Intern Med. 2017;167:714-724.

5. Kosche C, Mansh M, Luskus M, et al. Dermatologic care of sexual and gender minority/LGBTQIA youth, part 2: recognition and management of the unique dermatologic needs of SGM adolescents. Pediatr Dermatol. 2019;35:587-593.

6. Reiter PL, McRee A-L, Katz ML, et al. Human papillomavirus vaccination among young adult gay and bisexual men in the United States. Am J Public Health. 2015;105:96-102.

7. Charlton BM, Reisner SL, Agénor M, et al. Sexual orientation disparities in human papillomavirus vaccination in a longitudinal cohort of U.S. males and females. LGBT Health. 2017;4:202-209.

8. Agénor M, Peitzmeier SM, Gordon AR, et al. Sexual orientation identity disparities in human papillomavirus vaccination initiation and completion among young adult US women and men. Cancer Causes Control. 2016;27:1187-1196.

9. Loretan C, Chamberlain AT, Sanchez T, et al. Trends and characteristics associated with human papillomavirus vaccination uptake among men who have sex with men in the United States, 2014-2017. Sex Transm Dis. 2019;46:465-473

10. Setiawan D, Wondimu A, Ong K, et al. Cost effectiveness of human papillomavirus vaccination for men who have sex with men; reviewing the available evidence. Pharmacoeconomics. 2018;36:929-939.

11. Agénor M, Peitzmeier S, Gordon AR, et al. Sexual orientation identity disparities in awareness and initiation of the human papillomavirus vaccine among U.S. women and girls: a national survey. Ann Intern Med. 2015;163:99-106.

12. Benard VB, Castle PE, Jenison SA, et al. Population-based incidence rates of cervical intraepithelial neoplasia in the human papillomavirus vaccine era. JAMA Oncol. 2017;3:833-837.

13. McRee A-L, Gower AL, Reiter PL. Preventive healthcare services use among transgender young adults. Int J Transgend. 2018; 19:417-423.

14. Trinidad J. Policy focus: promoting human papilloma virus vaccine to prevent genital warts and cancer. Boston, MA: The Fenway Institute; 2012. https://fenwayhealth.org/documents/the-fenway -institute/policy-briefs/PolicyFocus_HPV_v4_10.09.12.pdf. Accessed September 15, 2020. 